\title{
Age of Onset of Physical Abuse: Implications for Adult Anger and Aggression
}

\author{
Stephanie D. Carter, ${ }^{1, *}$, Niki L. Crabtree ${ }^{2}$, James Epps ${ }^{3}$, Ann-Marie Roberts-Davis ${ }^{4}$ \\ ${ }^{1}$ Department of Psychology, Florida Institute of Technology, Melbourne, FL 32901 \\ ${ }^{2}$ Department of Education, University of South Florida \\ ${ }^{3}$ Department of Mental Health Law and Policy, University of South Florida \\ ${ }^{4}$ Private Practice in San Antonio, TX \\ *Corresponding Author: Scarter2012@my.fit.edu
}

Copyright @ 2014 Horizon Research Publishing All rights reserved.

\begin{abstract}
This study examines the impact the age of onset of physical abuse has on the expression of anger and aggression in adulthood. The Emotional and Physical Abuse Questionnaire (EPAB), State Trait Anger Expression Inventory (STAXI), Emotional Response Scenarios (ERS), and a demographics questionnaire were given to 229 undergraduate psychology students. Age of onset of abuse does predict higher levels of Trait Angry Temperament and Anger Expression Out in those whose abuse began as a child than for those whose abuse began in adolescence/ adulthood or those not abused. Further, it was determined that Hostile Attributional Bias moderated the relationship between age of onset of abuse and both Trait Angry Temperament and Anger Expression Out. The implications for the Social Information Processing theory of anger and aggression and potential therapeutic use of these findings are discussed.
\end{abstract}

Keywords Childhood Physical Abuse, Child Maltreatment, Anger, Aggression, Attributed Hostility

\section{Introduction}

A Social Information Processing (SIP) theory of anger and aggression, as reformulated by Crick and Dodge (1994), outlines the non-linear steps involved in enacting a certain behavior based on the perception and analysis of social stimuli. According to the model, the individual must first encode both internal and external cues, and then interpret those. Next, the individual must clarify his or her goals for the given situation. Following, one must access stored courses of action from his or her data base, or construct new responses if none are available. The individual then must evaluate and decide which response best fits the situation, and then proceed to enact the chosen behavior.

Response access and construction is a crucial step in the Social Information Processing model because many things can influence which responses are available or constructed.
Aspects such as emotions (Lemerise \&Arsenio, 2000), environment, and schemas can influence which responses are available for enactment. Schemas provide a blueprint for interpreting situational cues and formulating social responses to those cues. They are based in the individual's experience and become more salient over time, through rehearsal and practice. Schemas can be especially important when dealing with others because they can reduce reaction time by having a set of behaviors available quickly, instead of having to construct a new response from the beginning for each interaction. In the case of anger and aggression, this can lead to a preponderance of aggressive responses being available, while prosocial responses may go unconsidered.

The manner in which schemas also affect response decisions is detailed by Fontaine and Dodge (2006). A potential response must first meet a primary threshold of acceptability. To do so, the response must be generally relevant to the social interaction, situationally acceptable, and of sufficient internal congruence to the responding individual's self-concept. Theoretically, a response must meet each of these criteria in order to be considered for enactment, but the thresholds may vary according to situations. Behaviors that have been rehearsed and enacted many times and over various scenarios, i.e. schemas, may become habitual and pass the threshold quickly, or bypass it completely. Thus, events occurring early in life not only influence the construction of schemas, they also increase the probability of those schemas developing into automatic and inherent tendencies (Crick and Dodge, 1994). This is in keeping with the work of Elias and Berk (2002), who posit that the temperament of a child undergoes development until the age of three or four; therefore, the onset of abuse at a young age will affect the temperament of the child, possibly encouraging him or her to become more irritable and difficult to manage. Since temperament is a trait-like characteristic, rather than state-like, the temperament of a child will likely carry over into adulthood. Both formulations stress the disproportionate impact of events that occur at a young age.

One such schema-driven automatic tendency is Hostile 
Attributional Bias (Crick \&Dodge, 1994). This anger-related cognitive construct is a tendency to perceive hostile intent in the actions of others where social cues for actual intent are ambiguous. This is relevant to the stage of the SIP model in which environmental cues are interpreted. Such attributions prompt anger (a primarily emotional construct) and aggression (primarily behavioral). First evinced in children, Epps \& Kendall (1995) extended the construct to adults, and to not only ambiguous situations, but clearly benign and hostile ones as well. This biased cognitive process is thought to be a function of maladaptive, aggressive schemas.

Childhood physical abuse (CPA) produces schemas that promote and reinforce anger and aggression in future situations. Huesmann (1988) offers that aggressive behavior is learned through the enactive and observable acts of aggression throughout childhood. When a child is exposed to abuse, either as a witness or as a victim, the event will play a role in the development of schemas relating to anger and aggression. In the presence of relevant socio-behavioral cues, an individual brings these aggressive schemas to mind as a possible outcome or behavioral mechanism for the situation. It follows that those who experience physical abuse as a child have more opportunity to develop and rehearse schemas pertaining to abuse, anger, aggression, and violence compared to individuals who were physically abused only as an adolescent or adult and those who were not physically abused at all.

The present study examined the impact of age of onset of physical abuse on anger and aggression, and Hostile Attributional Bias (HAB). It was hypothesized that those whose physical abuse began as children will have higher scores on self-report measures of anger and aggression than those first abused as adolescents or adults or those who were not abused. Furthermore, it was hypothesized that HAB will moderate any significant relationships found between age of onset of abuse and anger/aggression variables.

\section{Methods}

\subsection{Procedure}

Along with a demographics questionnaire, participants were given a variety of other questionnaires. Of the surveys given, the ones pertinent to this particular study are the Emotional and Physical Abuse Questionnaire (EPAB), Emotional Response Scenarios (ERS), State and Trait Anger Scales (STAS), and the Anger Expression Inventory (AX).

\subsection{Participants}

A total of 229 participant based responses, as determined by the Emotional and Physical Abuse Questionnaire, were used in this study. All of the participants were enrolled in undergraduate psychology courses in San Antonio, TX and Tampa, FL. In exchange for their efforts, participants received extra credit that was applied to psychology classes.

\subsection{Instrumentation}

\subsubsection{The Emotional and Physical Abuse Questionnaire}

In order to assess history of physical abuse, participants completed the EPAB. The EPAB (Carlin et al., 1994) consists of 34 items that assess one's self-report of childhood discipline and abuse. Respondents use a 6-point Likert-type scale of frequency of occurrence $(0=$ "never," $5=$ "very frequently") to answer questions regarding specific childhood experiences. Thirteen items comprise the Physical Abuse subscale (PAB). Participants' patterns of responses to these items allow for a classification of physical abuse based upon an objective criterion. Specifically, if participants endorse any occurrence of eight major assault items (e.g., bones broken, purposefully burned) or respond with a frequency rating of " 3 " or greater to any of the five remaining items (e.g., pinched, shaken, hit with a stick), they are classified as having been physically abused. Additional questions ask participants to state whether or not they believe themselves to have been abused, and whether abuse occurred as a child, adolescent or adult. Three groups were derived from this measure: those subjects who denied a history of physical abuse $(n=186)$, those whose abuse began in childhood $(\mathrm{n}=26)$, and those whose abuse began in adolescence/adulthood $(\mathrm{n}=17)$. The EPAB also includes a subscale that measures emotional abuse. Although this subscale was administered, it was not be utilized in data analyses for the purposes of this study.

\subsubsection{Emotional Response Scenarios}

The ERS was modified for use in this study to assess angry reactions and hostile attributions to various situations. The original ERS consists of scaled responses to 22 scenarios that depict hypothetical interactions between the reader (i.e., the participant) and another person, the "protagonist" (Epps \& Kendall, 1995). Each scenario contains a "core" section and four "valence" statements. The core presents an introduction to the situation, offering details regarding the type of relationship between the protagonist and the reader, the condition that brings them into contact with one another, and any acts or words that pass between them. The core section offers ambiguous information related to the intent of the protagonist. The valence statements, on the other hand, contain information that allows the reader to attribute benign or hostile intent to the protagonist. Based upon the formula introduced by Dodge and Newman (1981), the ratio of hostile to benign valence statements is $3: 1$ for hostile scenarios, 1:3 for benign scenarios, and 2:2 for ambiguous scenarios.

Following Epps and Kendall's (1995) design, the 12 scenarios of the modified ERS were presented in randomized order two times, and participants answered different questions following each administration. During the first presentation, participants answered two questions regarding 
each scenario: (a) "How angry would you be if this happened to you?" and (b) "What would you do or say if this happened to you?" The phrase "say or do" was randomly replaced with "do or say" within each type of scenario. Answers to the anger question used a 9-point Likert-type scale ( 1 = "not at all angry," 9 = "extremely angry") and answers to the response question were open-ended and coded later for content. Participants then were asked to reread each scenario and answer two questions on 9-point Likert-type scales: (a) "How hostile do you feel the initiator's intentions were?” (1= "not at all hostile", 9 = "completely hostile"), and (b) "How angry would you be if this happened to you?" (1 = "not at all angry,” 9 = "extremely angry”). Participants were instructed to refrain from attempting to remember their initial responses when answering the second administration of the scenarios. The two sets of questions followed separate administrations of the scenarios in order to prevent possible contamination of anger ratings by the cognitive processing of attributions (Epps \& Kendall, 1995).

\subsubsection{The State-Trait Personality Inventory Trait Anger Scale}

In order to describe the relationship between anger as a dispositional characteristic and abuse status, participants completed the State and Trait Anger Scales of the State-Trait Personality Inventory (Spielberger, 1979). Although both scales were administered, only the Trait Anger scale was thought to be pertinent to the current study. The Trait Anger scale utilizes 4-point Likert-type frequency scales that are summed to indicate higher scores for higher characteristic anger. The Trait Anger scale is further subdivided into two subscales: Angry Temperament which measures an individual's general tendency to experience anger without provocation, and Angry Reaction which measures an individual's general inclination to experience anger when criticized or treated unfairly.

\subsubsection{Anger Expression Inventory}

The AX was used to describe the relationship between aggression and hostile attributions. The AX (Spielberger et al., 1985) is a 24-item self-report inventory comprised of three subscales that measure the frequency with which angry feelings are: suppressed (Anger Expression In), controlled (Anger Control), and expressed (Anger Expression Out). The subscale scores are combined to produce a general index of the frequency of anger expression, with higher scores indicative of greater aggression. Alpha coefficients ranging from .73 to .85 reflect good internal consistency for the AX subscales.

\section{Results}

Anger Expression Out. To test the hypothesis that the age of onset of abuse will significantly affect anger and aggression, Multivariate Analysis of Variance (MANOVA) was performed, with LSD post hoc testing. The independent variable, age of onset of abuse, had three levels: not abused, abused only as an adolescent or adult, and child onset abuse. The dependent variables were the Trait Angry temperament and Trait Angry Reaction scales and the scales of the AX. The MANOVA showed a significant multivariate effect (Hotelling's Trace: $F(10,442)=1.66 ; p=.035)$. Age of onset of abuse predicted Anger Expression Out $[\mathrm{F}(2,226)=4.37$, $p=0.014]$. The mean for those abused as a child $(M=17.73$, $\mathrm{SD}=4.54$ ) was significantly higher than for those abused as an adolescent or adult $\left(\mathrm{M}_{\mathrm{t}}=15.18, \mathrm{SD}=2.79\right)$ and those not abused $(M=15.22, S D=4.12)$. The latter two groups did not differ from each other. Age of onset of abuse also predicted trait angry-temperament $[\mathrm{F}(2,226)=4.51, \mathrm{p}=0.01]$. The mean for those abused as a child $(\mathrm{M}=7.73, \mathrm{SD}=3.58)$ was significantly higher than for those abused as an adolescent or adult $(\mathrm{M}=6.47, \mathrm{SD}=2.07)$ and those not abused $(\mathrm{M}=6.13$, $\mathrm{SD}=2.41$ ). The latter two groups did not differ from each other on either.

Hostile Attributional Bias.To test the hypothesis that the Hostile Attributional Bias moderates the relationship between age of onset of abuse and Trait Angry Temperament/Anger Expression out, it must be shown that (a) age of onset of abuse is related to both Trait Angry Temperament, and Anger Expression Out(demonstrated above); (b) that attributed hostility is related to Trait Angry Temperament, and Anger Expression Out; and (c) that age of onset continues to predict the Trait Angry Temperament and Anger Expression Out after the effects of attributed hostility have been accounted for (Baron and Kenny, 1985). To test the latter two requirements, Multivariate Analysis of Covariance (MANCOVA) was performed, with the three age-of-onset groups as the independent variable, attributed hostility as the covariate, and the two anger/aggression variables as dependent variables. The MANCOVA showed a significant multivariate effect (Hotelling's Trace: $\mathrm{F}(4,442)=3.50 ; \mathrm{p}=.008)$. A significant relationship between $\mathrm{HAB}$ and Trait Angry Temperament was first established [F $(1,213)=12.78, p=0.001]$. Next, the covariate, HAB, was determined to act as a moderator because the relationship between age of onset of abuse and Trait Angry Temperament remained significant after $\mathrm{HAB}$ was partialled out [F $(2,213)=5.41, \mathrm{p}=0.005]$.

Turning our attention to Anger Expression Out, a significant relationship between $\mathrm{HAB}$ and Anger Expression Out was first established [F $(1,213)=25.93, \mathrm{p}<0.001]$. Next, the covariate, $\mathrm{HAB}$, was determined to act as a moderator because the relationship between age of onset of abuse and Anger Expression Our remained significant after $\mathrm{HAB}$ was partialled out $[\mathrm{F}(2,213)=5.15, \mathrm{p}=0.007]$.

\section{Discussion}

The purpose of this study is to examine the impact that the age of onset of physical abuse has on anger and aggression in adulthood. Previous studies have determined there is a relationship between experiencing abuse as a child and 
becoming abusive in adulthood. It was hypothesized that individuals whose abuse began in childhood would have higher levels of anger and aggression than those who were never abused or whose abuse began in adolescence/adulthood. It was also hypothesized that the Hostile Attributional Bias would moderate the relationship between the age of onset of abuse and anger and aggression. Analysis of the results partially supported the first hypothesis: scores on the Anger Expression Out and Trait Angry Temperament subscales were higher among participants whose abuse began during childhood than for those who were never abused or whose abuse began in adolescence/adulthood. Additionally, the results fully supported the second hypothesis, in that the Hostile Attributional Bias moderated the relationship between age of onset of abuse and both Trait Angry Temperament and Anger Expression Out.

These findings support the influence of a hostile environment on the development of aggressive schemas, and the strengthening of the effect of those schemas with age. Individuals who have been exposed to physical abuse as a child will begin forming aggressive schemas concerning social interaction in times of conflict. The child will begin to associate the overt expression of anger with normal behavior, and this association will be reinforced the more it is experienced. On the other hand, individuals whose abuse does not begin until adolescence or adulthood may have spent a larger proportion of their developmental years building and maintaining typical, non-violent schemas; thus, they are able to differentiate normal behavior from abusive behavior, rather than view the two as indistinguishable. Consequently, the relationship between Trait Angry Temperament and Anger Expression Out and the age of onset of abuse was greater among those individuals whose abuse began in childhood, while those relationships did not between those whose abuse began in adolescence/adulthood and those who were never physically abused. As such, this supports the theories of Elias and Berk (2002) and of Crick and Dodge (1994).

The second hypothesis, that Hostile Attributional Bias would moderate the relationship between the age of onset of abuse and Trait Angry Temperament and Anger Expression Out, was supported by this study. That is to say, biases in attributed hostility are responsible for part of the relationship between age of onset of abuse and Temperament and outward expression of anger, but not the entire relationship. The establishment of $\mathrm{HAB}$, a cognitive construct, as a factor linking early childhood experience and self-reports of both emotion related and behavioral measure of adult anger/aggression provides much support for Social Information Processing views of the origin and maintenance of untoward behavioral tendencies.

There are some limitations in the present study regarding the sample. The sample size was relatively small, especially in the groups abused as children $(\mathrm{n}=26)$ and as adolescents $(n=17)$. Thus, type 2 error may underpin the dearth of findings linking the Age of Onset of Abuse and the remaining three subscales of the STAXI. A larger sample size could also increase our ability to generalize to a larger population. This study also utilized a convenience sample--all of the participants were undergraduate psychology students. This may be a particular problem in the study of aggression, as success in a university setting may employ a self-selection of individuals who exhibit more self-control or socially acceptable behavior. This may artificially truncate the range of response regarding anger and aggression. Further, the use of self-report measures may limit the validity of the relationships due to concerns regarding common method variance. Future research should strive for multimodal assessment.

This study expands on the current literature regarding the effects of childhood physical abuse. Not only did this study support previous findings that a history of physical abuse often results in higher levels of anger and aggression in adulthood, but it demonstrates that the timing of the abuse is an important factor for future behavior. In this study, the individuals whose abuse began as an adolescent or adult were statistically the same as those participants who were never abused on many anger/aggression variables. Understanding how abuse affects individuals at different ages can help tailor treatments given to abused children to help correct their maladaptive, aggressive schemas. The study also demonstrates the importance of addressing a Hostile Attributional Bias when treating victims of childhood physical abuse. Further research is needed to determine whether adult aggressive behaviors and angry temperaments among victims of childhood physical abuse may be altered by teaching a healthier attributional process.

\section{REFERENCES}

[1] Baron, R. M.\& Kenny, D. A. (1986). The moderator-mediator variable distinction in Social Psychological research: Conceptual, strategic and statistical considerations. Journal of Personality and Social Psychology, 51(6), 1173-1182.

[2] Carlin, A.S., Kemper, K., Ward, N.G., Sowell, H., Gustafson, B., \& Stevens, N. (1994). The effect of differences in objective and subjective definitions of childhood physical abuse on estimates of its incidence and relationship to psychopathology. Child Abuse and Neglect, 18, 393-399.

[3] Crick, N.R. \& Dodge, K.A. (1994).A review and reformulation of social information processing mechanisms in children's social adjustment. Psychological Bulletin, 115, 74101.

[4] Dodge, K.A.,\& Newman, J.P. (1981). Biased decision-making processes in aggressive boys. Journal of Abnormal Psychology, 90, 375-379.

[5] Elias, C. L., \&Berk, L.E. (2002). Self-regulation in young children: Is there a role for sociodramatic play? Early childhood research quarterly, 17 (2), 216-238.

[6] Epps, J., \& Kendall, P.C. (1995).Hostile attributional bias in adults. Cognitive Therapy and Research, 19, 159-178.

[7] Fontaine, R.G., \& Dodge, K.A. (2006). Real-time decision 
making and aggressive behavior in youth: A heuristic model of response evaluation and decision (RED). Aggressive Behavior, (32), 604-624.

[8] Huesmann, L.R. (1988). An information processing model for the development of aggression. Aggressive Behavior, 14,13-24.

[9] Lemerise, E.A. \&Arsenio, W.F. (2000).An integrated model of emotion processes and cognition in social information processing. Child Development, (71), 107-118.
[10] Spielberger, C.D. (1979). Preliminary Manual for the State-Trait Personality Inventory (STPI ).Unpublished manuscript. University of South Florida at Tampa.

[11] Spielberger, C.D., Johnson, E.H., Russell, S.F., Crane, R.S., Jacobs, G.A., \& Worden, T.J.(1985). The experience and expression of anger: Construction and validation of an anger expression scale. In M.A. Chesney \& R.H. Rosenman (Eds.), Anger and hostility in cardiovascular and behavioral disorders (pp. 5-30). New York: Hemisphere/McGraw Hill. 